\title{
Collisional Ion Energy Transfer Model for Understanding Wrinkles on Poly (dimethylsiloxane) Surfaces after Oxygen Ion Beam Irradiations
}

\author{
Seunghun Lee*, Junyoung Yang, Sunghoon Jung, Do-Geun Kim, and Eunyeon Byeon \\ Advanced Nano-Surface Department, Korea Institute of Materials Science, Gyeongsangnam-do 51510, Republic of Korea
}

Received September 28, 2018; revised October 18, 2018; accepted October 25, 2018

\begin{abstract}
We introduce a collisional ion energy transfer model to understand wrinkle wavelengths on poly (dimethylsiloxane) surfaces after ion beam irradiations. The collisional ion energy transfer model uses a Monte Carlo simulation and a full molecular method to predict the thickness of a hard skin layer produced by ion beam irradiations on poly (dimethylsiloxane) surfaces. Oxygen ion beam irradiations were conducted to verify the theoretical model. To make wrinkles with wavelength in the range of $0.6-1.0 \mu \mathrm{m}$, the model derived appropriate ion energies of 700-1,000 eV. Oxygen ion beams with the average energy of 707, 800, 901, and 1,043 eV were exposed on the polymer surfaces, which showed randomly distributed wrinkle patterns with wavelengths of $0.63 \pm 0.25,0.84 \pm 0.30,0.93 \pm 0.32$, $1.27 \pm 0.42 \mu \mathrm{m}$, respectively. The wavelengths observed through the experiments showed consistency with the collisional ion energy transfer model.
\end{abstract}

Keywords: Ion beam, Polymer, Wrinkle, Collision, Cross-linking

\section{Introduction}

Nanostructure surfaces on polymers are of considerable interest in nanotechnologies that include tunable optics [1], surface enhanced Raman spectroscopy [2], interlayer adhesion control [3], flexible electronics [4], and biomimetic micro fingerprints for anti-counterfeiting [5]. Wrinkle nanostructures on soft polymers have been intensively studied as wrinkle surface patterning is used in several applications such as stretchable hydrophobic substrates and security applications $[5,6]$. Wrinkle topologies have been fabricated spontaneously by means of mask-free processes, such as thin film deposition [7], plasma treatment [8], solvent-driven oxidation [9], thermal annealing [10], and ion beam (IB) irradiation [11]. These methods commonly form a hard skin layer that generates local moduli difference causing wrinkles [7]. In particular, the plasma treatment and ion beam irradiation using an ion bombardment is suitable to make the hard skin layer. Because ion-polymer surface interactions change the binding status within limited thickness below several tens $\mathrm{nm}$. In poly(dimethylsiloxane) (PDMS), the IB irradiation results in the formation of hard skin layer within 1-10 nm,

*Corresponding author

E-mail: seunghun@kims.re.kr which induces wrinkles with wavelength from hundreds $\mathrm{nm}$ to several $\mu \mathrm{m}$ because the wrinkle wavelength is a function of the thickness of hard skin layer [12].

Uniform wrinkle formation on PDMS substrates is important for industrial applications. Localized ion irradiation in focused ion beams showed the inhomogeneity of wrinkle patterns due to strain localization [11,13-15]. Large area IB irradiation with uniform ion flux and energy is necessary to make homogenous wrinkle patterns. A linear IB irradiation is possible to treat flexible substrates with a web speed of $1-10 \mathrm{~m} / \mathrm{min}$ when linear ion beam source and roll-to-roll treatment system are utilized [16]. The linear IB source emits linearly uniform gas ion beams such as argon, oxygen, nitrogen with a beam width of $1.5 \mathrm{~m}$ and an ion flux uniformity below $5 \%$. Thus, homogenous wrinkle formation is available in in-line or roll-to-roll processes without inhomogeneity problem due to locally induced strain after IB exposure. And the linear IB process is an attractive candidate to fabricate wrinkle surfaces with high throughput, relatively low cost, and clean process comparing to spin coating, electron beam lithography, optical lithography, and wet chemical processes.

The design of wrinkle wavelength is also important for the various applications. The parameters of IB irradiation such as ion energy and incident angle determine a depth in 
which the hard skin layer is formed. Recently, ion beam irradiations on PDMS elastomer have been reported to produce wrinkle structures as a function of the IB irradiation parameters $[12,17,18]$. The wrinkle wavelength has been modulated successfully in range of $0.75-1.95 \mu \mathrm{m}$ by changing IB energy from $800 \mathrm{eV}$ to $2000 \mathrm{eV}$ [18].

Also the wrinkle wavelength on PDMS have been controlled from $0.93 \mu \mathrm{m}$ to $2.03 \mu \mathrm{m}$ by adjusting the incident angle of impinging ions from $15^{\circ}$ to $90^{\circ}$. The ion incident angle changes cross-linking density, which affects the Young's modulus of the PDMS surface [12].

Understanding a wrinkle formation based on ionpolymer surface interactions is imperative to apply the IB technique to the wrinkle surface patterning. Many reports have introduced IB induced wrinkle patterns on PDMS with descriptions based on mechanical and physiochemical analysis, such as modulus measurement and X-ray photoelectron spectroscopy $[11,12,17]$. Though the postexperiment investigations explained wrinkle formation in detail, a theoretical model which predicts wrinkle formation is required to design a wrinkle wavelength for various purposes. In this work, we demonstrated quantitative interpretations of interactions between impinging ions and PDMS surfaces using a collisional ion energy transfer (CIET) model. The CIET model calculates a depth which is effective to form a the hard skin layer using Monte-Carlo simulations and a full molecular method [19,20]. The wavelength expected by the CIET model were compared with the wrinkle wavelength obtained after linear oxygen IB irradiation on PDMS surfaces.

\section{Experimental details}

PDMS precursor mixtures were prepared by a combination of Sylgard 184 base and hardener (Sylgard 184, Dow Corning Corporation, USA) in the mass ratio of 10:1, by mixing in a Petri dish for $10 \mathrm{~min}$. The prepared mixtures were placed in vacuum chamber, until no bubbles remained in the bulk of the mixtures. Glass substrates cleaned by a standard method were used for PDMS spin coating. The precursors mixtures were spin coated onto $25 \times 25 \mathrm{~mm}^{2}$ glass for $40 \mathrm{~s}$ at $500 \mathrm{rpm}$. This sample preparation procedure is identical to reference to use Young's modulus and Poisson's values of bulk PDMS in wavelength calculation [12]. The PDMS coatings were then annealed by heating on hotplate for $120 \mathrm{~min}$ at $65^{\circ} \mathrm{C}$. PDMS samples on the glass substrates were exposed to oxygen IB generated by closed drift ion sources [16]. The ion source emits linear IB (width: $400 \mathrm{~mm}$ ) with the ion current density of $0.1 \pm 0.02 \mathrm{~mA} / \mathrm{cm}^{2}$ and the ion dose irradiated on PDMS samples were $4.5 \pm 0.5 \times 10^{16} \mathrm{~cm}^{-2}$. The anode voltage controls the average ion energy: 707 , 800,901 , and $1,043 \mathrm{eV}$. A retarding potential analyzer (RPA) was used to measure the ion energy distribution function (IEDF) of ions irradiated on PDMS samples [21].
The IB irradiation was conducted in vacuum environment of 0.5-1 mTorr. The surface image of the wrinkle patterns was observed by field emission scanning electron microscopy (JSM-6700F, JEOL). The PDMS samples were prepared by $10 \mathrm{~nm}$ silver film coating using magnetron sputtering. The acceleration voltage was kept at $5 \mathrm{kV}$. The SEM images were subjected to 2D FFT analysis to characterize the wrinkle wavenumbers using commercial software $\left(\mathrm{SPIP}^{\mathrm{TM}}\right)$.

\section{Results and discussion}

Figure 1 demonstrates scanning electron microscope images and wavenumber distributions analyzed by twodimensional fast Fourier transform power density spectra. As the ion energy increases the PDMS surfaces showed a wrinkle width broadening, which corresponds to the decrease of wrinkle wavenumber: $1.60 \pm 0.56,1.19 \pm 0.38$, $1.08 \pm 0.34$, and $0.79 \pm 0.24 \mu \mathrm{m}^{-1}$ for each ion energy represented in Fig. 1(e). The deviations are the full width at half maximum of the wavenumber distribution. The

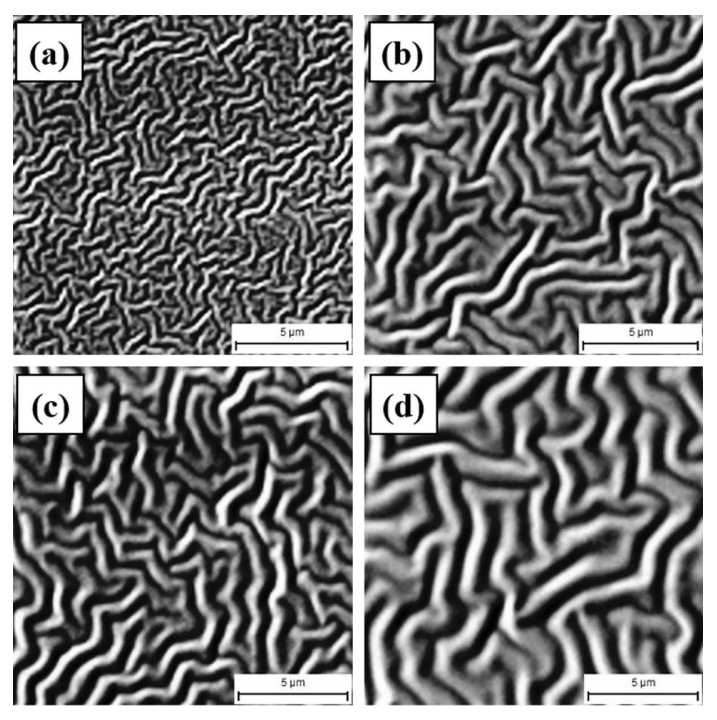

(e)

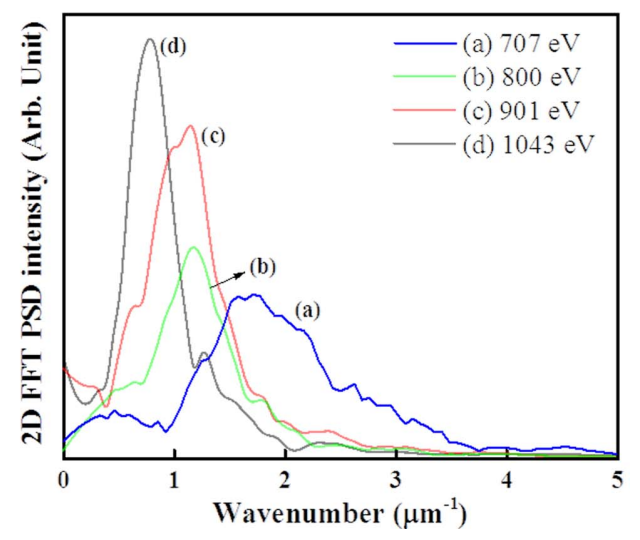

Figure 1. Surface images at the ion energy of (a) $707 \mathrm{eV}$, (b) $800 \mathrm{eV}$, (c) $901 \mathrm{eV}$, and (d) $1,043 \mathrm{eV}$, and (e) wavenumber distribution functions for each PDMS surfaces analyzed by $2 D$ FFT power density spectra. 


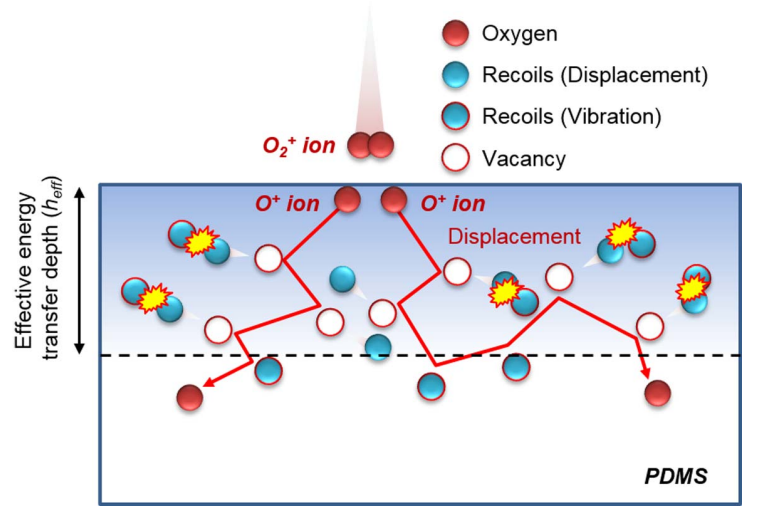

Figure 2. Schematic of collisional ion energy transfer model with full molecular method: oxygen molecule ion beam dissipated immediately to two monoatomic oxygen atoms at surface and modify PDMS surface within effective energy transfer depth.

wrinkle wavelengths that correspond to the wavenumbers are $0.63 \pm 0.25,0.84 \pm 0.30,0.93 \pm 0.32,1.27 \pm 0.42 \mu \mathrm{m}$, respectively.

IB irradiations make a hard skin layer that determines the wrinkle wavelength. The competition between the bending energy of the hard skin layer and the stretching energy of the soft supporting PDMS determines the wrinkle wavelength [17]. Eq. (1) including the Young's modulus and Poisson's ratio describes the competing behaviors:

$$
\lambda=4.4 \mathrm{~h}\left[\frac{E_{H S}\left(1-v_{P D M S^{2}}\right)}{E_{P D M S}\left(1-v_{H S^{2}}\right)}\right]^{1 / 3},
$$

where $\lambda$ is the wrinkle wavelength, $h$ is the thickness of the hard skin layer; $E_{H S}$ and $E_{P D M S}$ are the Young's moduli of the hard skin layer and the PDMS substrate, respectively; and $v_{H S}$ and $v_{P D M S}$ are the Poisson's ratios of the hard skin layer and the PDMS substrate, respectively. The Young's modulus of bulk PDMS has been controlled simply by annealing temperature [17]. In this work, the PDMS samples were annealed at $65^{\circ} \mathrm{C}$ for the Young's modulus of $1.5 \mathrm{MPa}$, which simplified Eq. (1) to $k=1 /$ $147 \mathrm{~h}$ using several known values, $E_{H S} \approx 70 \mathrm{GPa}, v_{H S} \approx 0.2$, and $v_{P D M S} \approx 0.48$ [12]. In this procedure, we assumed that the oxygen IB treated PDMS surfaces in Fig. 1 have same $E_{H S}$ and $v_{H S}$ values to predict wrinkle wavelength using the CIET model and Eq. (1).

Collisional behaviors between ions and polymers could explain the thickness of the hard skin layer. Irradiated ions penetrate polymer surfaces to a definite depth accompanying energy transfers by cascade collisions. Incident ions collide with polymer atoms, and make energetic recoils. Ions and recoils lose their energy to make displacements of polymer atoms: nuclear stopping. Displacement occurs when the transferred energy is larger than the threshold energy for displacement. Polymers have 2-10 eV displacement energy, which is smaller than that of semiconductors $(\sim 15 \mathrm{eV})$ and of metal $(25-40 \mathrm{eV})$ [22]. The displacement results in vacancy, replacement, and interstitial productions. If the transferred energy is not sufficient to generate displacement, phonon vibration consumes the recoil energy thermally [22]. The nuclear stopping is a driving force for scission and cross-linking initiating a formation of hard skin layers. Thus, quantitative estimation of the energy transfer as a function of depth could explain the thickness of the hard skin layer after IB irradiations.

The energy transfer as a function of depth was calculated by Monte Carlo based simulation. The stopping and range of ions in matter (SRIM) was used to predict collisional ion energy transfer rates to PDMS components: $\mathrm{C}, \mathrm{H}, \mathrm{O}, \mathrm{Si}$ [22]. In the SRIM calculation, the conventional PDMS formulation $\left(\mathrm{C}_{2} \mathrm{H}_{6} \mathrm{OSi}\right)_{\mathrm{n}}$ was adapted (density: $0.868 \mathrm{~g} /$ $\mathrm{cm}^{3}$ ). The calculation type was "detailed calculation with full damage cascades". Monoatomic IB irradiation, such as $\mathrm{He}^{+}$and $\mathrm{Ar}^{+}$ions, can be precisely calculated, because SRIM applies the binary collision approach. Meanwhile, molecular IBs, such as $\mathrm{O}_{2}^{+}$and $\mathrm{N}_{2}^{+}$, cannot be directly treated by binary collision. However, the penetration and energy loss of the molecular IBs can be calculated by using a theory of molecular IB implantations: the full molecular method [20]. This assumes that when it enters the target, the molecular ion dissipates immediately as shown in Fig. 1. This is true if the ion energy is much higher than the dissociation energy of molecular ions, which are of the order of $10 \mathrm{eV} /$ atom [20]. For ion energies in several hundreds $\mathrm{eV}$ range, this requirement for spontaneous dissociations is certainly met. Thus, an impacting molecular ion can be considered as two monoatomic ions. The incident energy is divided by the mass ratios represented in Eq. (2):

$$
\frac{E_{i}}{E}=\frac{M_{i}}{\sum_{j} N_{j} M_{j}},
$$

where, $E_{i}$ is the monoatomic ion's energy of $i$ species, $E$ is the energy of the molecular IB; $M_{i}$ and $M_{j}$ are the mass of each species, respectively; and $N_{j}$ is the atom number of $j$ species. In the molecular oxygen IB, the CIET model substitutes a molecular oxygen IB $\left(\mathrm{O}_{2}^{+}\right)$by two monoatomic oxygen atoms $(\mathrm{O})$ with half of the molecular ion's energy in PDMS medium. Actually, oxygen plasma generated by low temperature DC discharge consists of mostly molecular oxygen ions $\left(\mathrm{O}_{2}^{+}\right)[23,24]$. The few percent of monoatomic oxygen ions having a whole acceleration energy can be ignored in this experiment conditions. Thus, the CIET model assumes that molecular oxygen ions are dissociated to two monoatomic oxygen atoms, which have a half of molecular oxygen ion's energy.

The oxygen atoms transfer energy to recoils by collisions in Fig. 2. When the transferred energy to recoil is larger than the displacement energy of polymer components, the recoils induce vacancy, replacement, and interstitial to a certain depth, in which scission and cross-linking are occurred. When the transferred energy to recoil is lower than the displacement energy, recoils generate phonon that releases transferred energy thermally. The phonon in 
(a)
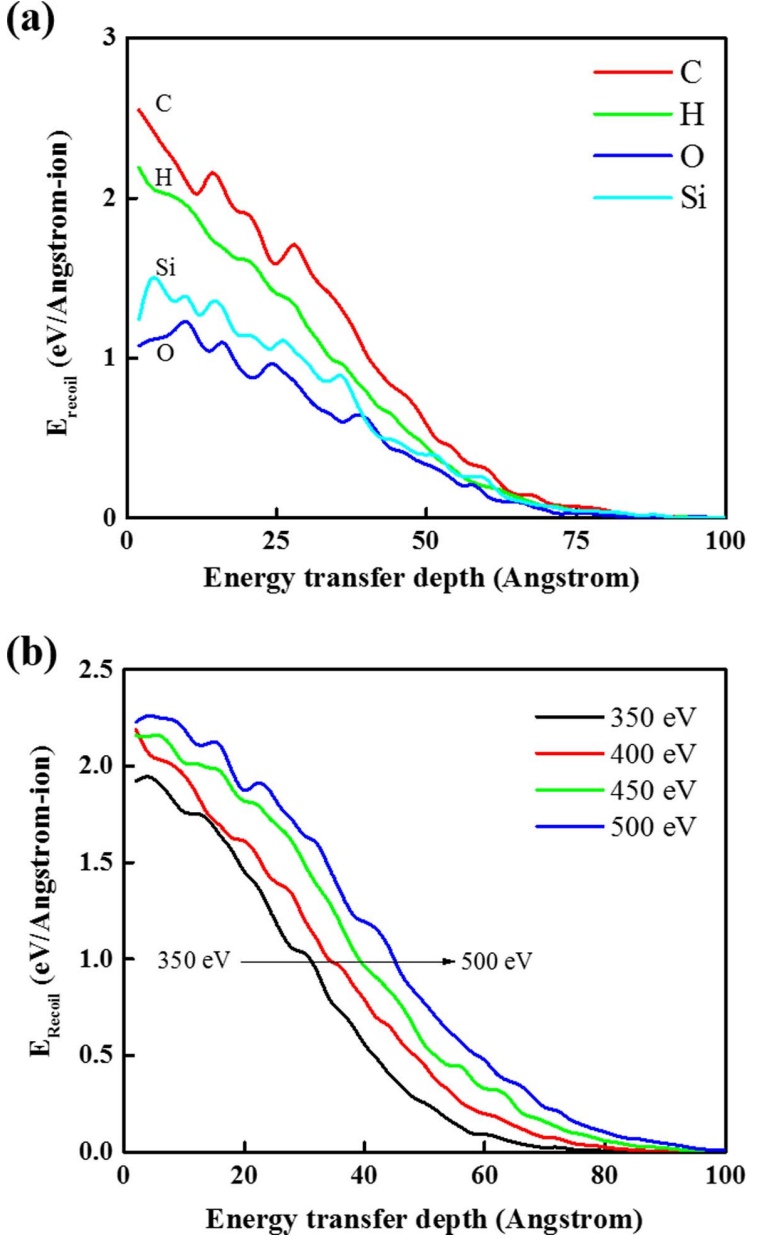

Figure 3. (a) Energy transfer rate to PDMS recoils (C, H, O, $\mathrm{Si}$ ) per unit depth by an atomic oxygen ion having an energy of $400 \mathrm{eV}$. (b) Energy transfer rate to hydrogen atoms per unit depth by an atomic oxygen ion with $350,400,450$, and $500 \mathrm{eV}$.

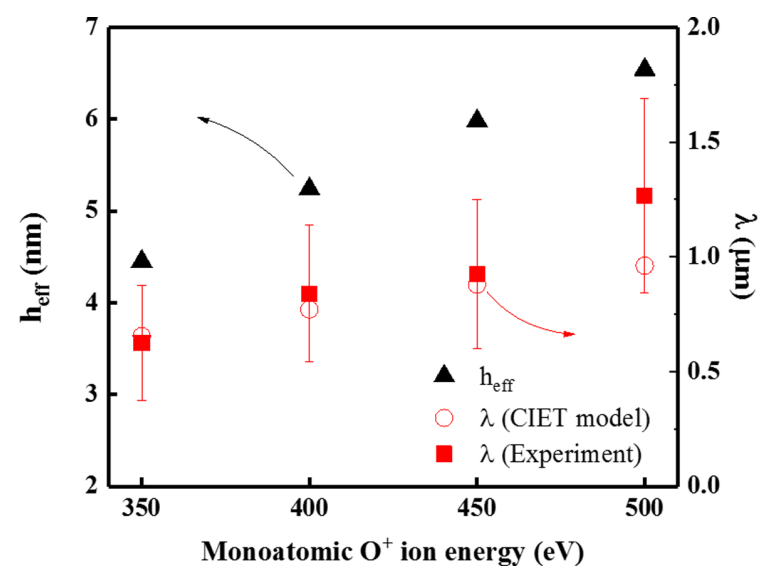

Figure 4. Effective energy transfer depth to dissociate a hydrogen from a methyl group, the corresponding wrinkle wavelength, and observed wavelength on PDMS surfaces as a function of the monoatomic oxygen energy. The hydrogen dissociation energy of $4.9 \mathrm{eV} / \mathrm{atom}$ was used in this plot.

amorphous phase cannot deliver heat efficiently and effective heat transfer length is lower than $10 \mathrm{~nm}$. The temperature increases locally in the ion energy transfer depth and the generated heat transfer with a low thermal conductivity of $0.1 \sim 0.2 \mathrm{~W} / \mathrm{m}-\mathrm{k}$ [25]. Therefore, it is necessary to consider the depth at which the transferred energy effectively change the binding of polymer surface due to displacement and heat generation. SRIM code calculates the collisional energy transfer rate to PDMS recoils $\left(E_{\text {recoil }}\right)$ of the monoatomic oxygen atom, which is used to calculate the effective energy transfer depth $\left(h_{\text {eff }}\right)$ in Fig. 1 creating hard skin layer after oxygen IB irradiations.

The $E_{\text {recoil }}$ was calculated by SRIM to predict $h$ in Eq. (1). Ions transfer their incident energy to the PDMS components: C, H, O, Si. Figure 3(a) shows the $E_{\text {recoil }}$ when the energy of atomic oxygen ion is $400 \mathrm{eV}$ at normal injection. Carbon and hydrogen atoms absorb ion energy dominantly at PDMS surface. Note that Methyl group $\left(\mathrm{CH}_{3}\right)$ dissociation is a main reaction to make the hard skin layer because the hard skin layer has no methyl group. For example, Sylgard 184 base PDMS is composed of dimethylsiloxane oligomers with vinyl-terminated end groups [26]. Pristine PDMS has shown strong infrared (IR) absorptions at 2,850 $\mathrm{cm}^{-1}\left(\mathrm{CH}_{2}\right.$ symmetric stretch), $2,910 \mathrm{~cm}^{-}$ ${ }^{1}\left(\mathrm{CH}_{3}\right.$ symmetric stretch), and $2,960 \mathrm{~cm} .{ }^{1}\left(\mathrm{CH}_{3}\right.$ asymmetric stretch). By oxygen plasma treatment, the methyl $\left(\mathrm{CH}_{3}\right)$ groups have been transformed into a hydroxyl-terminated surface with negligible residual methyl groups due to hydrogen dissociations [27]. Thus, we assume that the profile of $E_{\text {recoil }}$ to hydrogen atoms relates to the $h$ in Eq. (1).

Figure 3(b) shows the $E_{\text {recoil }}$ of an atomic oxygen to hydrogen atoms in PDMS medium as a function of impinging energy. The maximum energy transfer depth increased from $70 \AA$ to $100 \AA$ as the ion energy is increased from 350 to $500 \mathrm{eV}$, which corresponds to half of the molecular oxygen ion's energy, adapting the full molecular method represented in Eq. (2). Considering the hydrogen dissociation energy of a methyl chain in PDMS, monoatomic oxygen transfers enough energy to dissociate a hydrogen atom until a certain depth. We define the certain depth as an effective energy transfer depth $\left(h_{e f f}\right)$ that satisfies the following equation,

$$
E_{\text {diss }}=\int_{h_{\text {eff }}}^{\infty} E_{\text {recoil }}(H) d H,
$$

where $E_{\text {diss }}$ is the hydrogen dissociation energy, $h_{\text {eff }}$ is the effective energy transfer depth, $E_{\text {recoil }}$ is the energy transfer rate function in Fig. 3(b), and $H$ is the depth from the PDMS surface. Eq. (3) means that the $h_{\text {eff }}$ is the thickness when residual energy of penetrating oxygen atom is equal to the energy of methyl dissociation. In other words, $h_{\text {eff }}$ is the thickness at which a hydrogen dissociation of a methyl chain could be possible by energetic hydrogen recoils. For example, the hydrogen dissociation energy of a methyl chain is $473 \mathrm{~kJ} / \mathrm{mol}$, which is same to $E_{\text {diss }}=4.9 \mathrm{eV} /$ atom [28]. The $h_{\text {eff }}$ of $\mathrm{H}-\mathrm{CH}_{2}$ bonding as a function of the monoatomic oxygen ion energy from $350 \mathrm{eV}$ to $500 \mathrm{eV}$ is plotted in Fig. 4 . The $h_{\text {eff }}$ was obtained by integrating the 
plots of $E_{\text {recoil }}(H)$ in Fig. 3(b) within a range, which satisfies Eq. (3). It shows that monoatomic oxygen ions with energy of $350-500 \mathrm{eV}$ deliver sufficient energy to dissociate $\mathrm{H}-\mathrm{CH}_{2}$ bonding to the $h_{\text {eff }}$ of $45-65 \AA$. The dissociation depth is linearly increased due to that the $E_{\text {recoil }}$ of hydrogen atom is also linearly increased when monoatomic oxygen ion energy increases. Substituting $h$ in Eq. (1) to the $h_{\text {eff }}$ in Fig. 4, the wrinkle wavelength expected by $h_{\text {eff }}$ from the CIET model was $0.65-0.96 \mu \mathrm{m}$. The wrinkle wavelength observed in PDMS surfaces after oxygen IB irradiation in Fig. 1 showed consistency with the CIET model within the FWHM of wrinkle wavelength distribution. This shows the possibility that the amount of ion energy transferred to the PDMS recoils can explain the thickness of the hard skin layer using $h_{\text {eff }}$ based on the assumption that polymer bonds are dissociated when the binding energy is less than the energy delivered to the polymer components.

The prediction of wrinkle wavelength showed deviation in the oxygen ion energy of $500 \mathrm{eV}$. The PDMS specimens consist of a chain of Methyl $\left(\mathrm{CH}_{3}\right)$ groups $\left(\mathrm{E}_{\text {diss }}=3.5-4.9\right.$ eV/atom), Si-C ( $\mathrm{E}_{\text {diss }}=4.5 \mathrm{eV} /$ atom), Si-O $\left(\mathrm{E}_{\text {diss }}=8.3 \mathrm{eV} /\right.$ atom) [28]. As the energy of oxygen ion increases, the opportunity that ion beam irradiation initiates the dissociation of bonds that have higher $E_{\text {diss }}$ than that of Methyl groups increases, and it forms different deformed layer. Though the $h_{\text {eff }}$ in Fig. (4), which is predicted by the correlation between the $\mathrm{H}-\mathrm{CH}_{2}$ binding energy $\left(E_{\text {diss }}=4.9\right.$ $\mathrm{eV} /$ atom) and the $E_{\text {recoil }}$ to hydrogen atoms, showed reasonable wrinkle wavelength up to $400 \mathrm{eV}$, another dissociation reaction such as $\mathrm{Si}-\mathrm{C}$, $\mathrm{Si}-\mathrm{O}$ in PDMS should be included for precise wrinkle prediction at the higher ion energy above $400 \mathrm{eV}$.

\section{Conclusions}

Wrinkle wavelength predicted by the proposed collisional ion energy transfer model showed analogous values with the wrinkle wavelength observed after oxygen IB irradiations on PDMS surfaces. In the collisional ion energy transfer model, the effective energy transfer depth $\left(h_{\text {eff }}\right)$ for $\mathrm{H}-\mathrm{CH}_{2}$ bond dissociation is only considered to predict the thickness of hard skin layer. This approach showed consistency between the theoretical model and experimental result until a certain ion energy. When the ion energy increases and it causes double or triple dissociation processes, the collisional ion energy transfer model needs to deliberate the complex dissociation and cross-linking processes. In future work, improved CIET model including double or triple bond dissociation processes will be investigated for more precise expectation of the wrinkle wavelength after IB irradiation.

\section{Acknowledgements}

This work was supported by the National Research Foundation of Korea (NRF) funded by the Korean government (MSIP: Ministry of Science, ICT and Future Planning) (No. NRF-2017M2A2A6A01019037, and NRF2017M1A3A4A03025130).

\section{References}

[1] T. Ohzono and H. Monobe, Langmuir 26, 6127 (2010).

[2] T. Y. Jeon, S. Park, D. Kim, and S. Kim, Adv. Funct. Mater. 25, 4681 (2015)

[3] E. P. Chan, E. J. Smith, R. C. Hayward, and A. J. Crosby, Adv. Mater. 20, 711 (2008).

[4] D. Y. Khang, H. Jiang, Y. Huang, and J. A. Rogers, Science 311, 208 (2006).

[5] H. J. Bae, S. Bae, C. Park, S. Han, J. Kim, L. N. Kim, K. Kim, S. Song, W. Park, and S. Kwon, Adv. Mater. 27, 2083 (2015).

[6] W. K. Lee, W. B. Jung, S. R. Nagel, and T. W.Odom, Nano Lett. 16, 3774 (2016).

[7] N. Bowden, S. Brittain, A. G. Evans, J. W. Hutchinson, and G. M. Whitesides, Nature 393, 146 (1998).

[8] M. D. Huntington, C. J. Engel, A. J. Hryn, and T. W. Odom, ACS Appl. Mater. Interfaces 5, 6438 (2013).

[9] J. Xie, X. Han, C. Zong, H. Ji, and C. Lu, Macromolecules 48, 663 (2015).

[10] J. Y. Park, H. Y. Chae, C. H. Chung, S. J. Sim, J. Park, H. H. Lee, and P. J. Yoo, Soft Matter 6, 677 (2010).

[11] M. W. Moon, S. H. Lee, J. Y. Sun, K. H. Oh, A. Vaziri, and J. W. Hutchinson, Proc. Natl. Acad. Sci. 104, 1130 (2007).

[12] H. C. Jeong, H. G. Park, Y. H. Jung, J. H. Lee, B. Y. Oh, and D. S. Seo, Langmuir 32, 7138 (2016).

[13] Y. C. Chen and A. J. Crosby, Soft Matter 9, 43 (2013).

[14] V. Trujillo, J. Kim, and R. C. Hayward, Soft Matter 4, 564 (2008).

[15] M. W. Moon, S. H. Lee, J. Y. Sun, K. H. Oh, A. Vaziri, and J. W. Hutchinson, Scr. Mater. 57, 747 (2007).

[16] S. Lee, E. Y. Byun, J. K. Kim, and D. G. Kim, Curr. Appl. Phys. 14, S180 (2014).

[17] H. G. Park, H. C. Jeong, Y. H. Jung, and D. S. Seo, Sci. Rep. 5, 12356 (2015).

[18] H. C. Jeong, H. G. Park, J. H. Lee, and D. S. Seo, ACS Appl. Mater. Interfaces 7, 23216 (2015).

[19] J. F. Ziegler and J. P. Biersack, Treatise on Heavy-Ion Science (Springer, 1985), pp 93-129.

[20] A. Hössinger, PhD. Thesis, Technische Univ. Wien, 2000.

[21] C. Böhm and J. Perrin, Rev. Sci. Instrum. 64, 31 (1993).

[22] J. F. Ziegler, M. D. Ziegler, and J. P. Biersack, Nucl. Instruments Methods Phys. Res. Sect. B Beam Interact. with Mater. Atoms 268, 1818 (2010)

[23] P. F. Knewstubb, P. H. Dawson, and A. W. Tickner, J. Chem. Phys. 38, 1031 (1963)

[24] V. Vancoppenolle, P. Y. Jouan, M. Wautelet, J. P. Dauchot, and M. Hecq, J. Vac. Sci. Technol. A Vacuum Surfaces Film. 17, 3317 (1999).

[25] D. W. Van Krevelen, and K. Te Nijenhuis, Properties of Polymers (Elsevier, 2009).

[26] J. N. Lee, X. Jiang, D. Ryan, and G. M. Whitesides, Langmuir 20, 11684 (2004).

[27] H. Ye, Z. Gu, and D. H. Gracias, Langmuir 22, 1863 (2006).

[28] T. L. Cottrell, The Strengths of Chemical Bonds (Academic Press, 1958). 